\title{
The Effect of Potassium lodide Absorption on the Concentration of Antioxidants in Tomato Fruits
}

\author{
Medha Kini ${ }^{1}$ and Kate Flowers ${ }^{1}$ \\ ${ }^{1}$ Santa Clara High School, Santa Clara, California, USA
}

\begin{abstract}
Antioxidants are molecules found in fruits and vegetables that have the ability to fight substances in the human body that are detrimental to cells and genetic material. There is a high need for antioxidants, and the current method for boosting antioxidants is genetic modification, which is controversial in society; however, there is a lack of research concerning increasing antioxidant concentrations in plants in a natural manner. Thus, the efficacy of an iodine water drip can be called into question. Iodine has been proven to increase antioxidant enzymatic activity in animals and certain leafy vegetables. This paper will evaluate the effectiveness of an potassium iodide enriched water drip on the concentration of antioxidants in tomato plants. potassium iodide (KI) solution was created from diluting KI crystals into water, and the tomatoes were watered every day over a three-month growth period. At the end of the allotted growth period, four tomatoes were plucked from each group - the potassium iodide group, and the control group that was watered regularly. In order to calculate the increase in concentration, each tomato was mashed with an Efferdent denture-cleansing tablet so as to measure the absorbance of each sample using the blue dye from the tablet. Once obtaining the absorbance, the concentrations were found using Beer's law. Using a 2 sample T-test for the difference in antioxidants, the increase in antioxidants was found to be statistically significant.
\end{abstract}

\section{Introduction}

Often used as a marketing hook, antioxidants are molecules that have been known to benefit the human body since the 1990s, when scientists discovered the ability of substances to fight free radicals. Free radicals are substances that scavenge for electrons, able to damage cells and genetic material—and are produced by the human body as byproducts of turning food into energy (Arnarsan, 2019). Antioxidants, due to the fact that they are naturally extracted from food, have the capability to give electrons to free radicals in order to prevent oxidative stress.

The health benefits of antioxidants are greatly sought after by consumers. The compounds-namely, Vitamins $\mathrm{C}$ and $\mathrm{E}$ - have been referred to as "essential for the survival of all living things" by Harvard's School of Public Health. According to an article from the National Cancer Institute, the presence of exogenous antioxidants (antioxidants from food) have been shown to prevent the types of free radical damage that are associated with cancer development. A diet that includes antioxidants may reduce the risk of many other diseases, including heart disease, blood pressure, and diabetes.

Many consumers who have a need for antioxidants turn to supplements as a replacement for a healthy diet or professional medical care. The high doses of antioxidant concentration present in supplemental pills have been proven to be detrimental; they can interfere with medications, such as having a blood-thinning effect and interfering with the effectiveness of cancer treatment as well (Chun et al., 2016). The general advice is to avoid pills, and look towards produce instead. Thus, due to the high need for antioxidants, an area of research that has yet to be fully explored is the act of increasing antioxidant concentration in plants. 


\section{Literature Review}

\section{Current Methods}

In order to understand why this study analyzes how to increase antioxidant concentration in tomatoes, it is important to look at current research on the subject of antioxidants. The accumulation of increased healthy elements in farming crops would add immense value to the human diet, as well as increase crop yield and farm profit due to additional nutrients. This call to action is causing more and more scientists to turn their head towards improving agricultural efficiency. One example is, to make tomatoes healthier than before, researchers worldwide have turned to genetically engineering tomatoes with enhanced antioxidant properties.

In a study done by the University of Hong Kong and the Institut de Biologie Moléculaire des Plantes in France, a research group was able to enhance Vitamin E in tomatoes as well as double antioxidant concentrations in tomatoes. The group introduced a synthase, a type of enzyme, into the tomatoes. While there was no increase in size or appearance of the genetically modified tomatoes, the concentrations of certain pigments increased drastically-Bcarotene percentages increased by $169 \%$, and lycopene concentrations increased by $111 \%$. In turn, these concentrations exhibited $89.5 \%$ to $96.5 \%$ higher levels of antioxidants (HKU Press, 2017). This method was successfully able to promote Vitamin E as well as double B-carotene and lycopene contents in the genetically modified tomatoes-the research group also reported that the variant exhibited greater antioxidant activity in a separate experiment using Indian mustard plants that was referenced in the same study.

\section{Genetic Modification Controversy}

However, the controversy over genetic modification may outweigh the benefits. Despite the increased use of genetically modified (GM) food, many Americans know little about GM foods or hold ambivalent views on the issue. 39\% of Americans say that GM foods pose risks for one's health, and one-in-six Americans believe that GM foods are likely to bring environmental risks and health problems for the world population as a whole (Pew Research 2016). This public opinion and skepticism has a foundation in scientific findings as well.

There are many unknown consequences to changing the natural conditions of a plant, especially in regards to human safety-dangers of unintentionally introducing allergens into crops, risks of toxins, and widening the economic gap to name a few. Foreign gene expression has been proven to alter a plant's metabolism, rate of growth, and ability to adapt to environmental factors; this means that in addition to affecting the organism, the consequences influence the environment of the organism (Phillips, 2008). This might lead to ecological imbalances, such as allowing diseased plants to multiply without control, or exposure of toxic compounds into an ecosystem.

\section{Iodine Incorporation}

To solve this issue, this study aimed to approach plant growth in a less controversial and more accessible manner. There is a lack of research when it comes to boosting antioxidant concentration in plants through a natural method, especially one that can be used by an everyday home gardener. However, a study in the International Journal of Pharmacology found that iodine supplements functioned as antioxidants by increasing antioxidant enzyme activity in intoxicated rats. Results showed that oxidative stress in the rats, a prime factor in risk for cardiovascular disease, was neutralized by the supplementation of iodine in their diets (Agbor et al., 2011). The rats who received iodine supplements, in comparison to the control groups with no intoxication, had an increased antioxidant concentration of 89.73 $\mathrm{mol} / \mathrm{L}$ while the control group had an antioxidant level of $60.43 \mathrm{~mol} / \mathrm{L}$. The increase in antioxidant concentration paired with the decrease in oxidative stress suggests the fact that iodine has antioxidant potential, which can hold true even in plants.

Another study from the National Library of Medicine showed that iodine has been used to promote stress tolerance in aquatic plants, grains, and root crops; the application of iodine into agricultural practice has been proven to increase yield and growth in barley, beets, and radishes (Medrano-Macias et al., 2016). This increase in crop yield has been suggested to have been caused by the ability of iodine to be absorbed by plant roots and aerial plant structures, 
which have a great capacity to take unsaturated iodine in both the dissolved and gas forms. In addition to a strong correlation between enzyme activity in the crops and iodine absorption rate, the study explains that the peak of activity is during the reproductive stage of plant growth, also known as the flowering stage.

\section{The Tomato Plant}

In terms of choosing a plant, one of the most suitable candidates for iodine biofortification has been said to be tomatoes. This is mostly due to the tomato fruit's ability to withstand iodine stress; in an experiment outlined by Plantlab, Institute of Life Sciences, tomato plants resulted in being able to hold and accumulate great amounts of iodine in comparison to other fruits and vegetables such as spinach and lettuce. In addition to this quantitative study, the researchers from Plantlab also conducted a qualitative study to ascertain whether an increase of iodine affected fruit quality and visual appearance - the study concluded that there was no difference in size, shape, or color (Kiferle et al., 2013). It was determined that further research would need to be made concerning the interference of iodine with the primary compounds of the fruits themselves.

Iodine has often been used in tomatoes to prove abiotic and biotic tolerance-with the post-harvest biofortification of fruits like tomatoes, the absorption of iodine in the structure of the tomato plant is significant. Especially in the root of the tomato, iodine concentrations with a high degree of unsaturation can be taken in as an element from the soil as well as from the atmosphere (Medrano-Macias et al., 2016). The ability of the tomato plant to increase this tolerance of abiotic stressors is unique to the fruit. However, the fact that iodine absorption can occur through soil must still be tested to verify the iodine transfer from the tomato plants to organisms, which calls for additional examination.

Therefore, given the limitations of these previous studies, there is a gap in the body of knowledge concerning antioxidants consumed and natural factors that could boost antioxidants concentration in tomatoes. Research must be conducted to find a method that is accessible to the general public to increase the amount of antioxidants in plants, and if found, the results would be incredibly helpful for both everyday living as well as the agricultural world. In addition, there needs to be clarity on the connection between iodine enrichment and internal compounds within fruitbearing plants. This paper will attempt to bridge these gaps in research by asking the question, to what extent can a two-month growth period in potassium iodide enriched soil boost the concentration of antioxidants in fresh tomato fruits? Additionally, because no previous studies have been conducted in regard to these methods and their relationship with antioxidants in tomatoes specifically, the research will also fill a gap in knowledge on the subject.

\section{Methods}

The method chosen was an experimental method with one treatment. The experiment was started with two tomato plants, both in the flowering stage of growth-determined to be optimal by the National Library of Medicine. Rather than using iodine crystals, I decided to make a solution of potassium iodide (KI) to ensure that the concentration of KI could be controlled to keep the tomato plants healthy. The solution was 1 part per million potassium iodide. In order to create this exact molarity, one gram of crystals was diluted to 500 milliliters, stirred until dissolved. Then, 1.2 milliliters of this solution was extracted and diluted to 2400 milliliters.

$$
\begin{gathered}
1 \text { gram KI } / 500 \mathrm{~mL}=2000 \mathrm{ppm} \\
* * \text { needed: } 1 \text { ppm } \\
\text { Dilution equation: } m_{1} v_{1}=m_{2} v_{2} \\
2000 \mathrm{ppm}(1.2 \mathrm{~mL})=1 \mathrm{ppm}(\mathrm{x} \mathrm{mL}) \\
\mathrm{x}=2400 \mathrm{~mL}
\end{gathered}
$$

Using the dilution equation above, the proportions of volume of solution to concentration (parts per million) created a $1 \mathrm{ppm}$ solution. The threshold of 1 part per million was the average amount of iodine that tomato plants can withstand (Kiferle et al., 2013). 
The 1 ppm solution was re-diluted every three days, with the same volumes. One tomato plant was watered every morning with $500 \mathrm{~mL}$ of the $1 \mathrm{ppm}$ KI solution, and pruned weekly, while the other was the control and was watered regularly every morning without potassium iodide. The measurement of $500 \mathrm{~mL}$ is the recommended amount of water for one growing plant, mirroring approximately 1-2 inches of water in the pot. Both plants had multiple flowers primed for pollination. In the case of weather under 50 degrees Fahrenheit or extreme wind, the plants were covered and brought into a walled enclosure. At the end of the two-month growing period, four tomatoes were plucked from each plant. In order to determine the concentration of antioxidants in the plants, the study was specified to use a colorimeter - a machine that records light absorption — and an Efferdent tablet, which is a denture cleanser found at local pharmacies.

An increase in antioxidants can be measured both qualitatively and quantitatively. Using a saline solution made from $80 \mathrm{~mL}$ of water and 5 grams of salt, an Efferdent tablet was used to determine which plant had an increase in antioxidants. Because Efferdent tablets contain solid peroxide, adding an Efferdent tablet to a beaker containing a solution with antioxidants at the same time released a blue dye, which reacted with the antioxidants in the saline solution to produce a dark blue color. When dissolved in saline, monohydrate in the Efferdent tablet reacted to form hydrogen peroxide. The hydrogen peroxide then reacted with a catalyst in the tablet to form oxygen and water. The reaction was observed for around 20 minutes, and the change in blue dye intensity was recorded afterwards. As stated in an article from the Journal of Nutrition and Food Sciences, "the darker the remaining blue color of the solution tested, the more antioxidant activity is associated with the sample".

This qualitative analysis of antioxidant concentration allowed for a colorimeter to be used to quantitatively calculate the concentration of antioxidants based on the color of the solution. To create a standard unit of antioxidant activity, a sample of pure antioxidant was used. A beaker was first filled with $80 \mathrm{~mL}$ of distilled water and $500 \mathrm{~g}$ of ascorbic acid, Vitamin C. An Efferdent tablet was added and stirred until dissolved. The solution was then used in a colorimeter to calculate maximum absorbance at a certain wavelength. The absorbance calculated was then used to calculate sample concentration of antioxidants for the remaining tomatoes, assuming a concentration of 1 for ascorbic acid.

Once calculated, using eight glass beakers, $80 \mathrm{~mL}$ of deionized water was placed into each. The four tomatoes were plucked from each of the two tomato plants that had already been growing, one in potassium iodide enriched soil and one in regular potting soil, creating eight test samples. Each tomato from the potassium iodide enriched treatment and the potting soil control group was mashed into each separate beaker, removing tomato skin and cleaning supplies in between each sample so as to prevent contamination between samples. Once mashed, one Efferdent tablet was added to each sample, and stirred thoroughly.

Each sample was left to incubate for 25 minutes, and the resulting blue color of the Efferdent coloration from each sample was measured and compared against the maximum absorbance of the $500 \mathrm{~g}$ ascorbic acid solution. From each sample, the absorbance was recorded by a colorimeter. The cuvette was cleaned with deionized water between each trial. To compare the absorbance quantitatively, Beer's Law and the definition of absorbance was used to calculate the concentration of antioxidants in the treated tomatoes versus the control group of tomatoes. Beer's Law can be defined as $\mathrm{A}=\varepsilon b c$, where A stands for absorbance, $\varepsilon$ is molar absorptivity, and $\mathrm{b}$ is the path length of the sample, usually expressed in $\mathrm{cm}$. For this experiment, the colorimeter has a path length of $1 \mathrm{~cm}$, and molar absorptivity can be calculated from the original ascorbic acid solution using the concentration of 1 and the calculated value of absorbance.

\section{Results}

The colorimeter readings that measured absorbance for each sample gave the absorbances of the eight tomato-efferdent solutions. Each sample was tested three times, and averaged to get the sample absorbance reading used for calculating concentration. After recording the absorbances of both the potassium iodide enriched tomatoes and the control group of tomatoes, the absorbances were plotted on a side by side bar chart and analyzed in detail. The order of the tomatoes graphed had no influence on the comparison of the data. Beer's Law was rearranged to yield an equation 
used to relate the variables of absorbance and concentration. Using the proportions in Beer's Law as outlined below, the concentration of ascorbic acid was able to be related to the absorbances of the tomatoes, and the concentrations of the antioxidants in tomatoes were thus also able to be graphed side by side.

$$
\mathrm{A}=\varepsilon \mathrm{bc} \rightarrow C_{\text {antioxidant }}=\frac{A_{\text {antioxidant }} \cdot C_{\text {ascorbic }}}{A_{\text {ascorbic }}}
$$

Example calculation: control group of tomatoes sample 1

$$
C_{\text {antioxidant }}=\frac{0.321 \cdot 1}{1.7}=0.1889
$$

Calculating antioxidant concentration in the remaining samples were completed in a similar fashion. The concentration of ascorbic acid that was used for each concentration was always 1, as it was purely antioxidant from the original test used to calculate maximum absorbance. The concentration shown as an example yielded an absorbance of 1.7 at the given wavelength $635 \mathrm{~nm}$, and was substituted accordingly into the equation to solve for the sample concentration. There were no outliers in the data that were significant.

\section{Graphs and Raw Data}

\section{Absorbances of Tomato/Efferdent solution}

0.8

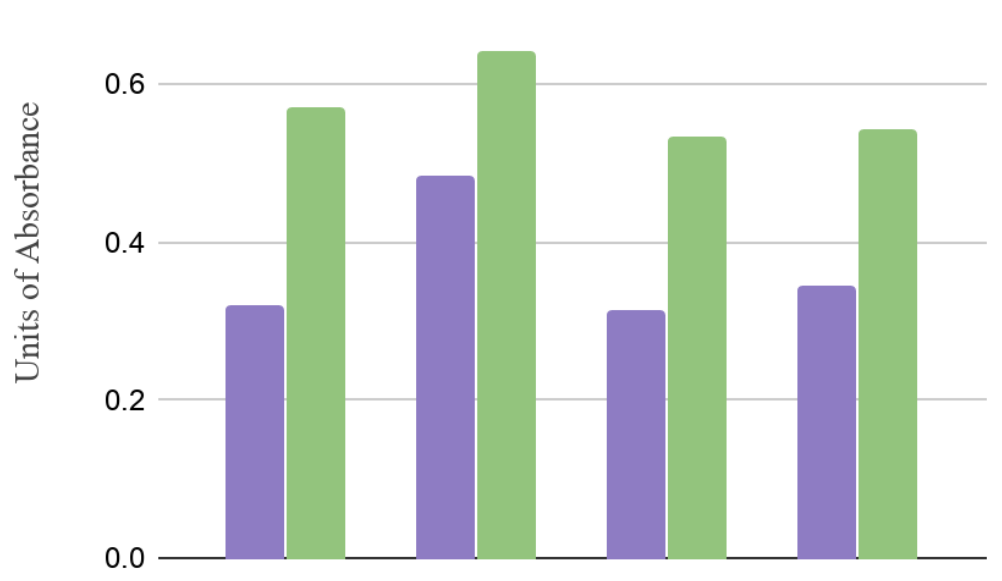

Control

KI Enriched

Figure 1. Side-by-side bar chart of tomato/Efferdent solution absorbance 


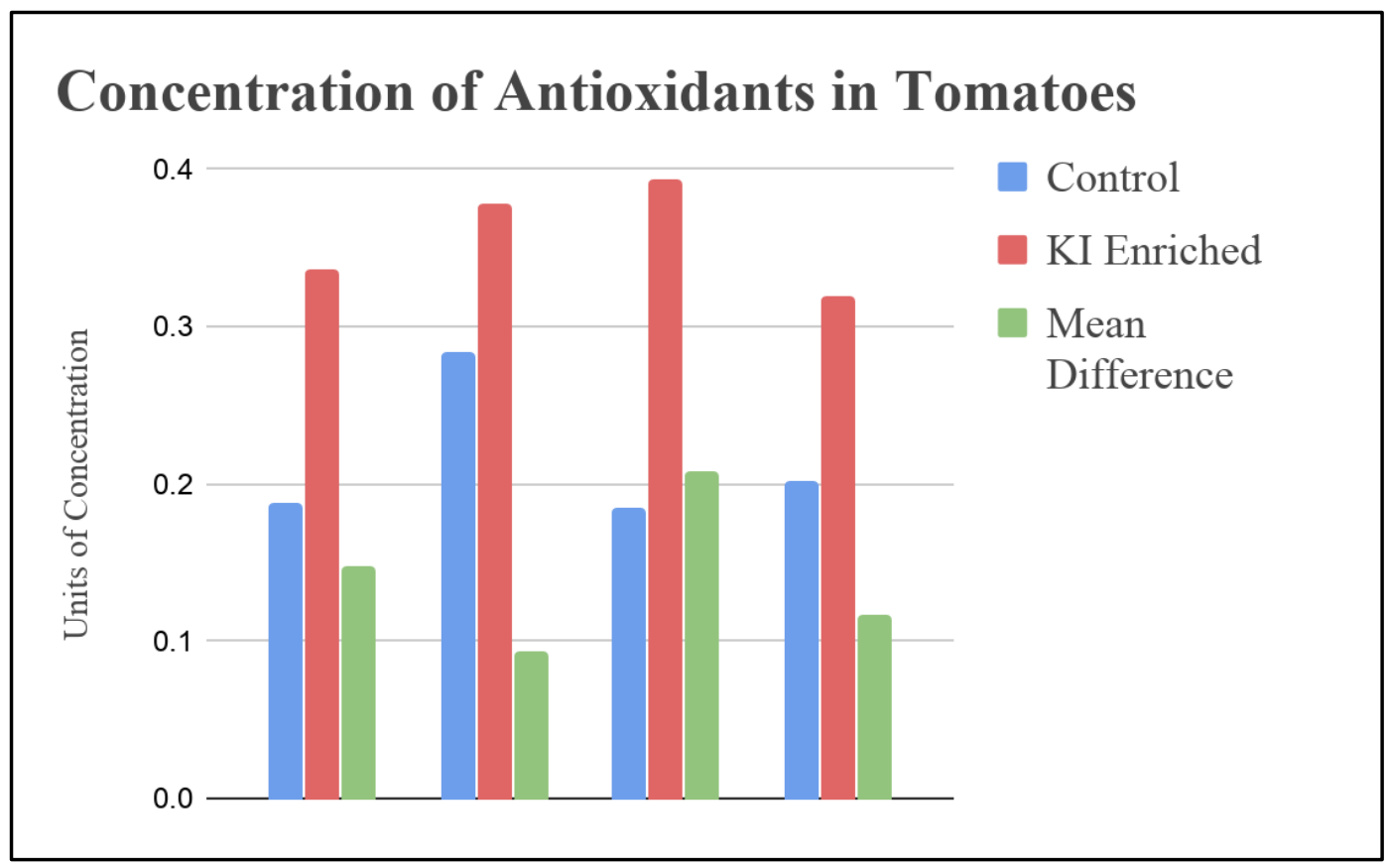

Figure 2. Side-by-side bar chart of antioxidant concentration and mean difference

\begin{tabular}{|c|c|c|c|c|c|}
\hline & Absorbance & \multicolumn{2}{|c|}{} & Concentration & \\
& Control & KI Enriched & & Control & KI Enriched \\
\hline $\begin{array}{c}\text { Average Ab- } \\
\text { sorbance: }\end{array}$ & 0.36575 & 0.57325 & $\begin{array}{r}\text { Average } \\
\text { Concentration: }\end{array}$ & 0.215147 & 0.337206 \\
\hline $\begin{array}{c}\text { Median } \\
\text { Absorbance: }\end{array}$ & 0.3325 & 0.558 & $\begin{array}{r}\text { Median } \\
\text { Concentration: }\end{array}$ & 0.195576 & 0.357059 \\
\cline { 1 - 1 } $\begin{array}{c}\text { Absorbance } \\
\text { Range: }\end{array}$ & 0.168 & 0.107 & $\begin{array}{r}\text { Concentration } \\
\text { Range: }\end{array}$ & 0.098823 & 0.07411 \\
\hline
\end{tabular}

Figure 3. Raw data table comparing absorbance and concentration of groups 


\section{Significance Tests}

$\mu_{c}=$ true mean antioxidant concentration in control group of tomato fruits

$\mu_{i}=$ the true mean antioxidant concentration in potassium iodide enriched tomato fruits

$\alpha=0.05$

$H_{0}: \mu_{i}-\mu_{c}=0$

$H_{a}: \mu_{i}-\mu_{c}>0$

Procedure: 2 sample T-test for $\mu_{i}-\mu_{c}$

Conditions :

- Random: tomato plants were randomly assigned treatments

- $10 \%: 4$ is less than $10 \%$ of all tomatoes

- Normal/large sample: $4 \leq 30$. However, only two samples are needed to declare normality. Based on the graph of concentration, normality can be determined. Therefore, all conditions are met.

After eliminating the possibility of outliers and checking the conditions of significance tests, a 2 sample T-test for $\mu_{i}-\mu_{c}$ was performed to determine the significance of the results against a 0.05 significance level, based on the difference between the mean concentration of antioxidants in the control group and potassium iodide enriched group of tomatoes. A 2 sample T-test for difference of means was used because groups were formed using completely randomized design, and the data came from two independent random samples. The null hypothesis, when subtracting control from experimental, was that the true mean difference in antioxidant concentration is equal to zero, and the alternative hypothesis was that the difference is greater than zero, as the hypothesis inferred an increase in concentration. The mean concentrations $(\bar{x})$ for the control group and KI enriched group were 0.2151 and 0.3372 respectively, and the standard deviations $\left(S_{x}\right)$ of the two groups were 0.0466 and 0.0285 .

$$
\begin{aligned}
& \text { Test statistic: } t=\frac{\left(\bar{x}_{i}-\bar{x}_{C \cdot i}\right)-\left(\mu_{i}-\mu_{C}\right)}{\sqrt{\frac{s_{i}^{2}}{n_{i}}+\frac{s_{C}^{2}}{n_{C}}}}=\frac{(0.3772-0.2151)-0}{\sqrt{\frac{0.0285^{2}}{4}+\frac{0.0466^{2}}{4}}} \\
& =\frac{0.1221-0}{\sqrt{2.031 \cdot 10^{-4}+5.429 \cdot 10^{-4}}}=\mathbf{4 . 4 7 1 ,} \boldsymbol{d} \boldsymbol{f}=\mathbf{3}
\end{aligned}
$$

The p-value that resulted from this T-test was $0.00334<\alpha=0.05$. The p-value can be interpreted as if assuming there is no difference in the mean difference in antioxidant concentration between control and KI enriched tomatoes, there is approximately a $0.334 \%$ chance of getting a difference of 0.1221 or more by chance alone. Thus, the null hypothesis, $H_{0}$, is rejected. From this, it can be concluded that there is convincing evidence that antioxidant concentrations in the KI enriched tomatoes were higher. Therefore, it is clear that the differences in antioxidant concentration between the data sets were significant, and the KI enriched soil was able to have a significant influence on the level of antioxidants in the experimental group of tomatoes.

\section{Discussion}

As seen in Figures 2 and 3, the antioxidant concentrations of the tomatoes that were watered with potassium iodide solution were, on average, higher than that of the control group tomatoes at the end of the two-month growing period. This was due to the fact that the calculated absorbances were higher for the potassium iodide enriched tomatoes. This suggests that iodine can have a considerable influence on antioxidant concentration; further analysis revealed that the p-value of this data set was lower than the chosen significance level of 0.05 , concluding that the averages between the potassium iodide enriched tomatoes and the control group of tomatoes are significantly different, though only just.

Thus, due to the significance between the experimental and control tomatoes, the initial alternative hypothesis was accepted: potassium iodide enriched soil does have a significant effect on antioxidant concentration in tomatoes. 
However, this conclusion must be taken with caution. Having understood that iodine, a widely used fertilizer for increasing the activity of enzymatic antioxidants, was successful in boosting antioxidant concentration, the implications of these findings can now be discussed. It is now clear that a natural method to boost internal compounds in plants is possible and proven to work. Firstly, these results show that watering plants with elemental solutions can function as an alternative to controversial methods, such as genetic modification. Secondly, the significant difference in antioxidant concentration paves the way for future research involving different methods and test subjects, in order to embrace natural methods of making the world healthier.

\section{Limitations}

In light of accepting the initial hypothesis, there are limitations that need to be mentioned. The biggest challenges concerning this research had to do with weather/time of year and human measurement error. It is difficult to grow tomatoes in the winter, thus the two-month growth period resulted in fewer tomatoes than needed to successfully determine a greatly significant increase in antioxidant concentration. The four tomatoes that were plucked off of each of the plants were weighed to ensure similar mass and size, in order to theoretically have similar antioxidant concentrations amongst the tomatoes. However, that wasn't the case-even amongst the control group, there were significant differences between each tomato's antioxidant concentration. This can be attributed both to the non-optimal season and weather for tomato growth, which would have influenced the importance of the "control" tomato group when it came to comparing antioxidant concentrations. However, this limitation is mitigated due to the fact that one can expect differences between the tomatoes, and a similarity in size or color may not translate to a similarity in antioxidants. For instance, a larger tomato compared to a smaller tomato treated with an iodine drip may not have a greater concentration of antioxidants simply due to its size in comparison, which was an assumption that was made in this experiment when picking the four tomatoes from each treatment group.

Another possible limitation is human error in measurement. When creating the potassium iodide solution, many precise measurements had to be taken in order to create the exact molarity of potassium iodide. There were multiple dilutions that were performed, such as the starting dilution of potassium iodide solution that then had to be diluted to 1 part per million (ppm) every three days. Because of the number of times that measurements had to be taken, it is possible that the concentration of potassium iodide was different per each dilution-many liquid measurements were taken by the use of a graduated cylinder that had values incrementing by milliliter; a more ideal measuring tool would have been increasingly more precise.

In addition, the absorbances that were used to calculate the concentration of antioxidants were measured using the tomato and Efferdent solution. This was done due to the fact that the blue dye in the Efferdent tablet is proportional to the amount of antioxidants in a certain substance. It is possible that the proportion of Efferdent dye to antioxidant concentration was not consistent amongst the tomatoes. This would have led to a false positive of an increase in antioxidants. The desired wavelength to compare the antioxidant concentration in tomatoes to the ascorbic acid values was $610 \mathrm{~nm}$ as stated in the procedure taken by the Journal of Nutrition and Food Sciences, but the colorimeters available for use did not have that exact wavelength (Al-Shahrani et al., 2013). The wavelength used for this experiment was $635 \mathrm{~nm}$, which resulted in some fluctuation between the absorbances. This could have led to a lesser observed experimental value for absorbance for both the control and potassium iodide enriched groups, but should not have had a significant effect when comparing the difference between the two.

\section{Future Research}

Further study into methods of increasing antioxidant concentration in plants should address the limitations above, by repeating the experiment on a larger scale, with the right weather conditions for growing the desired plants and consistent replicability. With the correct equipment and temperature control, the majority of the limitations from the current research are taken care of. Specific comparisons and accurate conclusions can be drawn from a larger pool of 
samples, and may be able to suggest statistically significant results. Sampling accuracy can be increased by incorporating more tomato samples, or extending the research to other plants.

For instance, the implementation of a concentrated form of iodine as plant nutrients can also be applied to plants other than tomatoes with high levels of antioxidants, such as broccoli, carrots, spinach, and potatoes. The differences in antioxidant concentration will be able to concede which plants are most greatly affected by an iodine boost, which would in turn have greater implications for homeowners and the farming industry. An experiment such as this can also test more combinations of antioxidant molecules with other aspects of plant nutrition, such as increased fiber and low saturated fats-both attributes that are typically related to antioxidant concentrations in plants. Another future direction is to verify whether this increase can be found in future generations of plants. There are two possibilities to confirm: either a child plant from the parent line with increased nutrient density will have to continue to be watered with solution to achieve these high nutrient results, or the child will have the desired nutrient content without the continued use of solution.

In addition, more research can be done concerning specific forms of antioxidants present in plant fruits such as lycopene and glutathione, which can be measured separately rather than as a general increase in enzymatic antioxidants as was done in this experiment. Another avenue for research could incorporate increases in compounds other than antioxidants - this can be done by correlating iodine content with sodium, magnesium, calcium, and other content of common minerals. Through biofortification methods and iodine incorporation, an increase in biomass and mineral composition can also be measured based on the iodine applied to the soil.

\section{Conclusion}

The results of this study point to the effectiveness of iodine in increasing antioxidant concentration in tomatoes-the difference between the control group of tomatoes and the experimental group was calculated to be statistically significant. However, when taking the limitations into account, further studies comparing the effectiveness of an iodine solution into tomatoes should be conducted. Therefore, future research should concern ideal weather conditions, specific and accurate experimental tools, and especially methods of comparing Efferdent absorbance to antioxidant concentration. In addition, it must be discerned whether this increase in antioxidant concentration will be inherited in future generations of the tomato plants. The results of these potential future studies would be able to determine whether a potassium iodide solution and incorporating other natural elements as fertilizer is an effective method to increase antioxidants in tomatoes, thus ascertaining a strong alternative to current unethical and unnatural methods of boosting antioxidant concentration.

\section{Acknowledgments}

I would like to thank Ms. Kate Flowers for helping me with this project.

\section{References}

Agbor, G. A., Taga, I., Nguindex, D. R., Zaidi, M. A., Lehman, L. G., Altosaar, I., \& Ngogang, J. Y. (2011). Effect of Iodine Supplementation on Antioxidant Status of Normal and Alloxan Monohydrate in Toxicated Rats. International Journal of Pharmacology, 7, 726-731. https://scialert.net/fulltextmobile/?doi=ijp.2011.726.731.

Al-Shahrani, M., Ahman, G. S., \& Amanullah, M. (2013). Measurement of Antioxidant Activity in Selected Food Products and Nutraceuticals . Journal of Nutrition and Food Sciences, 3(3). https://www.longdom.org/open-access/measurement-of-antioxidant-activity 
-in-selected-food-products-and-nutraceuticals-2155-9600.1000205.pdf

Antioxidants. (2019, November 14). Retrieved from https://www.hsph.harvard.edu/nutritionsource/antioxidants/

Arnarson, A. (2019, July 29). Antioxidants Explained In Simple Terms. Retrieved from https://www.healthline.com/nutrition/antioxidants-explained

Chun, O., Frei, B., \& Gardner, C. (2016, May 4). Antioxidants and Cancer Prevention. Retrieved from https://www.cancer.gov/about-cancer/causes-prevention/risk/diet/antioxidants-fact-sheet

$H K U$ researchers generate tomatoes with enhanced antioxidant properties by genetic engineering. (2017, November 9). Retrieved from https://www.hku.hk/press/news detail 17055.html

Kiferle, C., Gonzali, S., Holwerda, H. T., Ibaceta, R. R., \& Perata, P. (2013, May 31). Tomato fruits: a good target for iodine biofortification. Retrieved from https://www.frontiersin.org/articles/10.3389/fpls.2013.00205/full

Medrano-Macias, J., Lejia-Martinez, P., Gonzales-Morales, S., Juarez-Maldonado, A., \& Benavides-Mendoza, A. (2016, August 23). Use of Iodine to Biofortify and Promote Growth and Stress Tolerance in Crops. https://www.ncbi.nlm.nih.gov/pubmed/27602033.

Phillips, T. (2008). Genetically Modified Organisms (GMOs): Transgenic Crops and Recombinant DNA Technology. Retrieved from https://www.nature.com/scitable/topicpage/genetically-modified-organisms-gmostransgenic-crops-and-732/

Public opinion about genetically modified foods and trust in scientists. (2019, December 30). https://www.pewresearch.org/science/2016/12/01/public-opinion-about-genetically-modified-foods-andtrust-in-scientists-connected-with-these-foods/ 